ISSN: 2788-6506

Editada por: Instituto Universitario de Innovación Ciencia y Tecnología Inudi Perú<smiles>[CH]1C[CH]C1</smiles>

\title{
Vínculos afectivos y su influencia en el desempeño académico
}

Affective links and their influence on academic performance

Links afetivos e sua influência no desempenho académico

\author{
Liliana Muñoz ${ }^{1}$ \\ Institución Educativa Rural San Antonio (Colombia) \\ Dora Romo \\ Instituto Colombiano de Bienestar Familiar (Colombia) \\ Natalye Bravo \\ Diócesis Mocoa Sibundoy (Colombia)
}

DOI: https://doi.org/10.35622/j.rep.2021.01.004

Recibido 02/07/2019/ Aceptado 20/11/2020

RESUMEN. Este artículo aborda los factores protectores y de vulnerabilidad que impactan en el éxito o fracaso escolar. El objetivo principal es caracterizar los vínculos afectivos vivenciados en las familias para establecer su influencia en el desempeño académico, en una población escolar de 8 a 11 años. Para ello, se implementó una investigación con enfoque cualitativo y diseño fenomenológico, por estar centrado fundamentalmente en explorar, describir y comprender las experiencias familiares y como estas inciden en el desarrollo integral de los niños y niñas en sus dimensiones afectiva, social, cognitiva y su impacto en los procesos de aprendizaje.

PALABRAS CLAVE: Desempeño académico, factores protectores, familia, vínculo afectivo.

ABSTRACT. This article addresses the protective and vulnerability factors that impact school success or failure. The main objective is to characterize the affective bonds experienced in families to establish their influence on academic performance in a school population aged 8 to 11 years. For this, an investigation with a qualitative approach and phenomenological design was implemented, since it was fundamentally focused on exploring, describing and understanding family experiences and how they affect the integral development of children in their affective, social, cognitive and their dimensions impact on learning processes.

KEYWORDS: Academic performance, protective factors, family, affective bond.

RESUM0. Este artigo aborda os fatores de proteção e vulnerabilidade que afetam o sucesso ou o fracasso escolar. 0 objetivo principal é caracterizar os vínculos afetivos vivenciados nas famílias para estabelecer sua influência no desempenho acadêmico, em uma população escolar de 8 a 11 anos. Para tanto, foi realizada uma investigação com abordagem qualitativa e desenho fenomenológico, uma vez que se centrou fundamentalmente em explorar, descrever e compreender as experiências familiares e como estas afetam o desenvolvimento integral da criança em suas dimensões afetiva, social, cognitiva e suas dimensões. processos de aprendizagem.

${ }^{1}$ Correspondencia: liligoticamunoz@gmail.com 
PALABRAS CLAVE: Desempenho acadêmico, fatores de proteção, família, vínculo afetivo.

\section{INTRODUCCIÓN}

En la educación, la afectividad cumple un papel fundamental en el proceso de aprendizaje y desarrollo de habilidades de los estudiantes, convirtiéndolos en personas más activas, receptivas e integrales. Al respecto, Morgan (2006), afirma que el término afecto influye de manera directa en el comportamiento, los sentimientos, creencias, emociones, y actitudes. Es así como en la investigación se concibió la pertinencia de abordar las dimensiones: Familiares y educativas, ahondando en las relaciones afectivas, con el propósito de avanzar en la comprensión del tipo de vínculo afectivo construido en la relación paternofilial asociada al nivel de desempeño académico.

Así lo plantean Morón, Pérez y Pedrero, al referir que:

Los principales factores de protección en el ámbito de la familia tienen que ver fundamentalmente con la capacidad de desempeñar dos tareas críticas: la de ejercer una adecuada supervisión de la conducta de los hijos, y la de ofrecer relaciones afectivas positivas y apoyo emocional (2017, p.53).

Además, Morón et ál., exponen que:

la familia es la base en el fomento valores, actitudes, comportamientos prosociales, comunicación y escucha activa, así mismo de la relevancia de establecer límites y normas claras, como también brindar un clima familiar afectivo de reconocimiento y aprobación que facilite la expresión de emociones favorecedores de estabilidad emocional (2017, p.53).

\section{METODOLOGÍA}

\section{a) Diseño}

Para caracterizar los vínculos afectivos y su nivel de influencia en el desempeño académico, el estudio se desarrolló mediante un enfoque cualitativo con diseño fenomenológico bajo el paradigma socio constructivista, el cual está centrado fundamentalmente en explorar, describir y comprender las experiencias de los sujetos de estudio y encontrar los elementos en común, con el fin de identificar el grado de afectividad construida al interior de las familias y su nivel de incidencia en el desempeño académico de 14 estudiantes en edad infantil. Como estrategia Investigativa se empleó el método de estudio de caso, soportado por Yin (1994) que lo define como una "indagación empírica que utiliza múltiples fuentes de conocimiento para investigar un fenómeno actual dentro de su contexto de vida real", lo anterior a fin de garantizar la fiabilidad y validez de la investigación, que implicó realizar un 
examen minucioso del entorno y la realidad en la que están inmersas las unidades de análisis (p.46).

\section{b) Población y muestra}

La población objeto de estudio estuvo conformada por un total de 109 estudiantes del centro educativo rural San Antonio del Porotoyaco, distribuidos en seis sedes multigrado, dos ubicadas en zonas de difícil acceso en las veredas de Minchoy y Patoyaco, y las cuatro restantes cerca de la cabecera municipal: San Isidro, San José del Chunga, la Cabaña y la sede central San Antonio del Porotoyaco, ubicada al suroriente del municipio de San Francisco Putumayo. La muestra definida, según Hernández et al (2010), es "un subconjunto de elementos que pertenecen a ese conjunto definido en sus características, al que se llama población" (p.65). La presente investigación está representada por 14 estudiantes (12 familias) de los grados tercero, cuarto y quinto de la sede central San Antonio del Porotoyaco.

\section{Criterios de inclusión}

La elección de los casos se realizó de forma propositiva, es decir, con fundamento en las necesidades del estudio, el contexto y las posibilidades de acceso a la información; al respecto, no existe una indicación específica, referente al número de casos que deben hacer parte de la investigación, en tanto que el investigador tiene autonomía para hacer la selección e incluir los casos que considere, por tratarse de una investigación cualitativa (Perry, 1998). Así, con respecto a la presente investigación, se seleccionó como unidades de análisis la muestra descrita en la población, teniendo en cuenta que una de las autoras es docente de la citada institución y tiene relación directa con el 100\% de los sujetos de estudio.

\section{c) Consideraciones éticas}

Es pertinente resaltar que, la investigación educativa está regulada bajo una normatividad y atiende las consideraciones éticas, para el presente estudio se destaca la resolución 8430 del 4 de Octubre de 1993, la cual en sus artículos contiene un amplio soporte que la fundamenta, se resalta el artículo 5 que, establece como prioridad de todo sujeto de estudio el respeto a la dignidad humana, sus derechos y bienestar; de la misma forma el artículo 6, menciona que la investigación deberá cumplir con los principios éticos, y científicos que la justifiquen. Por lo anterior, exige que la forma en que se utilizan los datos debe ser responsable, ética y reservada, tanto su metodología, temas de estudio como en los instrumentos aplicados; además el contenido de los artículos 14 y 15 describe la importancia de indagar el interés individual de los sujetos en participar de la investigación, de comunicar los objetivos y alcance del estudio investigativo, a su vez la formalización con la autorización y firma del consentimiento informado. Finalizando, responsablemente el estudio con la divulgación de los resultados a los participantes. Atendiendo la normatividad y el derecho que tienen los participantes de un estudio a la confidencialidad y el anonimato (Hernández, 
Fernández y Baptista, 2014), no se mencionan los nombres de los participantes entrevistados "la confidencialidad es absoluta" (p. 511).

\section{RESULTADOS}

Con la información sistematizada se establecieron una serie de parámetros, los cuales se utilizaron con el fin de agrupar los datos recolectados, de tal forma que su análisis sea de fácil comprensión y como resultado de ese proceso, se establece la categoría axial. Lo que conllevó a relacionar la pregunta problematizadora, los instrumentos que se aplicaron para la recolección de la información y las subcategorías de análisis emergentes.

Tabla 1. Matriz de codificación de estudiantes

\begin{tabular}{|c|c|c|c|}
\hline Cod. & Nombre Estudiante & Código Estudiante & Gradc \\
\hline 1 & Estudiante 01 & 01Est & 3 \\
\hline 2 & Estudiante 02 & 02Est & 4 \\
\hline 3 & Estudiante 03 & 03Est & 5 \\
\hline 4 & Estudiante 04 & 04Est & 5 \\
\hline 5 & Estudiante 05 & 05Est & 5 \\
\hline 6 & Estudiante 06 & 06Est & 3 \\
\hline 7 & Estudiante 07 & 07Est & 5 \\
\hline 8 & Estudiante 08 & 08Est & 4 \\
\hline 9 & Estudiante 09 & 09Est & 5 \\
\hline 10 & Estudiante 10 & 10Est & 3 \\
\hline 11 & Estudiante 11 & 11Est & 4 \\
\hline 12 & Estudiante 12 & 12Est & 4 \\
\hline 13 & Estudiante 13 & 13Est & 3 \\
\hline 14 & Estudiante 14 & 14Est & 4 \\
\hline
\end{tabular}

Fuente. Elaboración propia 
Tabla 2. Matriz de codificación de padres de familia

\begin{tabular}{cc}
\hline Código Estudiante & Nombre Padre de Familia \\
\hline 01Pad & Padre de Estudiante 01 \\
02 Pad & Padre de Estudiante 02 \\
03 Pad & Padre de Estudiante 03 \\
04 Pad & Padre de Estudiante 04 \\
05 Pad & Padre de Estudiante 05 \\
06 Pad & Padre de Estudiante 06 \\
07 Pad & Padre de Estudiante 07 \\
08 Pad & Padre de Estudiante 08 \\
09 Pad & Padre de Estudiante 09 \\
10 Pad & Padre de Estudiante 10 \\
11 Pad & Padre de Estudiante 11 \\
\hline Padre de Estudiante 12
\end{tabular}

Fuente. Elaboración propia

\section{a) Recolección de los datos}

Las técnicas que se utilizaron en la investigación para la recolección de los datos fueron: la observación directa, documentos y registros (notas y seguimiento del estudiante) y la entrevista semiestructurada practicada a estudiantes, padres de familia y docente que permitieron la obtención de los datos e información directamente de la realidad. Para ello se tomó el consentimiento informado de las 14 familias intervenidas.

El contexto familiar es entendido como el espacio ideal para garantizar la fiabilidad de los datos y además permite hacer una confrontación entre lo que se dice y se observa en las relaciones de sus miembros. La aplicación de las técnicas de recolección de información se 
realizó a través de visitas a las familias en horarios previamente acordados.

Para Hernández et al (2014), la observación es un método sistemático de recolección de datos observables de los actores, disponiendo especial interés y atención a eventos, sucesos y detalles en la exploración del actuar, dialogar, y comportar dentro de sus comunidades, culturas, entornos, ambientes, y aspectos de vida, generando hipótesis para futuras investigaciones e identificando problemáticas (p.399).

En este sentido, la observación que se implementó fue cualitativa selectiva, orientada a estudiar los aspectos más significativos evidenciados en las relaciones que se dan entre los miembros de la familia en su contexto.

Las entrevistas semiestructuradas estuvieron encaminadas a explorar y caracterizar los vínculos afectivos de los estudiantes del aula multigrado de tercero, cuarto y quinto, padres y docente, a través de un dialogo sobre temas relacionados con los procesos de aprendizaje. La entrevista, según Sampieri (2014), cuenta con dos actores el entrevistador y entrevistado y consiste fundamentalmente en una guía de preguntas predefinidas flexibles, que el entrevistador lleva preparadas y que son aplicadas de forma presencial dialogada y amistosa, centrada en la interacción sobre experiencias anecdóticas, historias, de vida, percepciones, sentimientos, emociones, hechos, creencias, valores y opiniones (p.403)

El guion de la entrevista semiestructurada se elaboró con base en la revisión literaria contenida en el marco teórico del presente documento, este fue diseñado previamente siguiendo un guion temático con preguntas abiertas, encaminadas a dar respuesta a los objetivos propuestos teniendo en cuenta las siguientes categorías:

\section{Comunicación}

Vygotsky hace una contribución importante al mencionar que la comunicación como factor de mediación es de carácter indispensable para el aprendizaje y por ende el desempeño académico se ve influenciado en la medida que se dé la interacción social.

\section{Desempeños Académicos}

El Paradigma Constructivista asume que el conocimiento es una construcción mental resultado de la actividad cognoscitiva del sujeto que aprende. Concibe el conocimiento como una construcción propia, que surge de las comprensiones logradas a partir de los fenómenos que se quieren conocer.

\section{Empatía}

Hace referencia a las habilidades cognitivas, emocionales o afectivas del individuo, en la cuales este es capaz de ponerse en la situación emocional de otro.

\section{Factores de Vulnerabilidad}

Entendida como carencia de apoyo socio familiar, violencia física, psicológica o intrafamiliar, 
negligencia, falta de recursos económicos, pautas de crianza inadecuadas, falta de tiempo, comunicación agresiva o pasiva, tipología familiar y desinterés en rutinas escolares.

\section{Factores Protectores}

Definida como modelos individuales o de relación, actitudes, conductas, circunstancias individuales y colectivas, que se van conformando en un medio social (familiar en el caso de los niños) y que incluyen aspectos relacionados con la salud, educación, vivienda, afectos y conductas sanas y saludables.

\section{Rutinas Escolares}

Actividades que pueden contribuir a la generación de ambientes de aprendizajes, en los cuales se rescatan aspectos sociales, emocionales, cognitivos y relaciones familiares.

\section{Tiempo}

El tiempo es un concepto amplio que se aplica en diversos contextos. El tiempo puede ser visto como la magnitud física que permite secuenciar hechos y determinar momentos.

\section{Tipología familiar}

Estructura de la composición familiar:

- Familia nuclear.

- Familia extensa.

- Familia monoparental.

- Familia reconstituida.

- Familia de padres separados.

Se seleccionaron los factores comunicación, empatía, tiempo, factores protectores, factores de vulneración y tipología familiar, debido a que, en el ámbito académico, dichos aspectos constituyen factores asociados al paradigma constructivista de las teorías del aprendizaje.

De esta forma, la entrevista semiestructurada, quedó compuesta por 15 preguntas, de las cuales, tres (3) aluden a la comunicación; una (1) al tiempo, tres (3) a la categoría de rutinas escolares, dos (2) se refieren a la empatía; mientras que las seis (6) ultimas corresponden a los factores de protección y/o Vulneración.

\section{Documentos y registros.}

Para Sampieri (2014), los datos cualitativos son una fuente extremadamente valiosa como lo son los diferentes soportes documentales, equipos tecnológicos (cámaras, celular), registros (videos, fotografías) materiales que contribuyen al entendimiento del fenómeno social objeto de estudio (p.418). Para el investigador es de especial interés conocer las vivencias y los antecedentes de las personas, comunidades, o grupos. Por ello fue importante documentar el presente estudio con registros que cuenta la institución educativa 
frente al historial en el archivo de las notas, seguimientos y observador del estudiante, ficha sociodemográfica etc. Esta información permite conocer los antecedentes, las vivencias y los cambios registrados a lo largo del periodo académico, se alude de igual forma a grabaciones realizadas por medio de teléfonos celulares, tabletas y otros dispositivos que permiten, entre otras cosas, entender lo que pudo ocurrir, en qué periodo y cuáles fueron las experiencias, hechos, consecuencias y reacciones de las personas.

Así mismo el resultado del desempeño académico del promedio general de notas que obtuvieron los estudiantes durante el año lectivo, promedio considerando las nueve (9) áreas obligatorias estipuladas en la ley 115 y que están en escala de 1 a 5 puntos, con desempeños: bajo, básico, alto y superior.

\section{b) Análisis de los datos}

La Investigación cualitativa tiene como característica fundamental el trabajar principalmente con palabras y no con números, por lo tanto, el proceso que se realizó para agrupar la información obtenida en categorías se hizo asociando las palabras por su nivel de proximidad o afinidad también llamada "codificación", posteriormente se realizó el proceso de triangulación de los resultados obtenidos con el fin de develar subjetividades y respuestas al objetivo de la investigación

En este sentido el software NVIVO12 fue una herramienta de apoyo muy importante para obtener información más confiable frente al estudio realizado, debido a que posee un diseño capaz de procesar información cualitativa especialmente útil para el análisis de datos no estructurados como videos, audios, fotografías, textos, testimonios y bibliografías (QSR International, 2014), el programa ejecuta potentes herramientas para relacionar dicha información a partir de conceptos generadores, los cuales estructuran vinculaciones para análisis y establecer relaciones con la teoría, por lo anterior se hizo necesario codificar la información correspondiente a cada unidad de análisis (sujetos de estudio) de acuerdo a sus relatos y experiencias.

\section{Codificación abierta}

Según Hernández, Fernández, y Baptista (2014) este proceso corresponde a la revisión detallada de la información, y su clasificación. Específicamente, esta técnica consiste en desagregar meticulosamente la información para analizarla, compararla, determinar los elementos que presentan similitudes conceptuales y ameritan posteriormente una agrupación común, bajo una determinada denominación (p.427). La selección y construcción de códigos se hizo a partir de las características y significados, propios de la investigación (Strauss y Corbin, 2002). De esta manera, se abordó el texto correspondiente a la transcripción de las entrevistas practicadas. Se examinó línea a línea el texto de cada entrevista, se identificaron elementos relevantes para cada caso, los cuales se desagregaron y posteriormente se agruparon. Lo anterior, con el fin de obtener información capaz de representar el contexto que fue analizado.

Con los resultados de las entrevistas semiestructuradas incorporadas al software y con base 
a los informes consolidados que emite esta herramienta, se procedió a codificar y agrupar la información de acuerdo con factores comunes, categorías que se soportan y contienen a las unidades de análisis a través de una codificación abierta.

Así, los resultados concretos de la codificación abierta fueron: "códigos construidos" a partir de la interacción, frecuencia y cercanía de las palabras como se puede evidenciar en el reporte que emite el programa en las figuras 1 y 2.

Figura 1. Grafo circular resultado de los testimonios de las entrevistas semiestructuradas

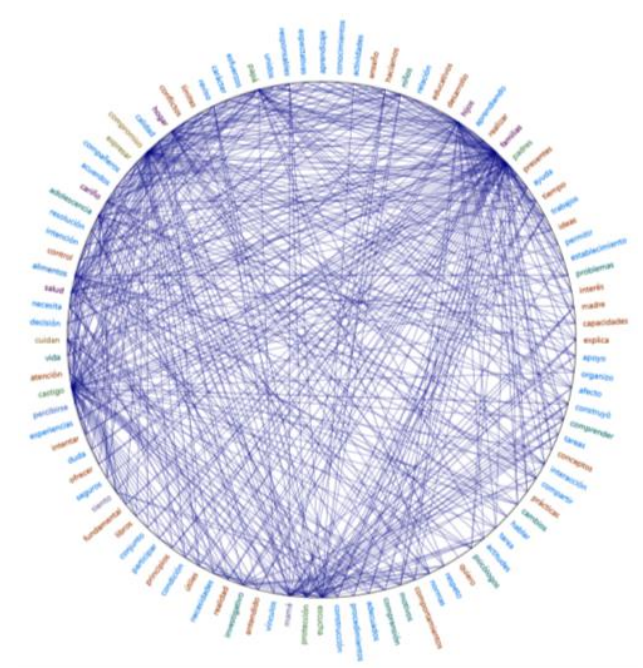

Nota. El grafo circular permite identificar la cercanía e interacción que existe entre algunas palabras posibilitando la categorización y agrupación para su correspondiente análisis. Elaboración Propia. Apoyo herramienta cualitativa NVIVO12.

Figura 2. Nube de palabras

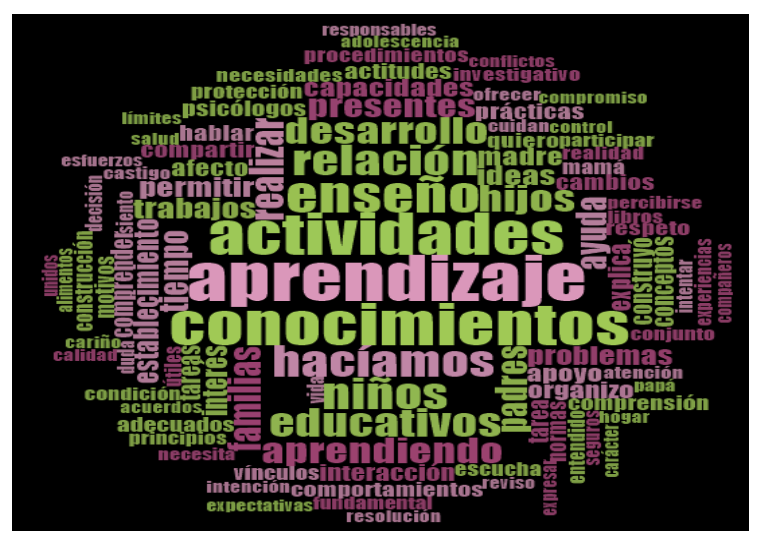

Nota. La nube posibilita visualizar de manera general las palabras más frecuentes a partir de los testimonios resultados de las entrevistas semiestructuradas practicadas a catorce (14) estudiantes, doce (12) padres de familia y una (1) docente. Elaboración propia. Apoyo herramienta cualitativa NVIVO12.

\section{Codificación axial.}

A partir de los resultados de la codificación abierta, y mediante un proceso de agrupación Liliana Muñoz, Dora Romo; Natalye Bravo 
se establecieron unas categorías relevantes, las cuales se denominaron "códigos sintetizados" (Hernández, Fernández, y Baptista, 2014, pág. 689). Al respecto Quintana (2006) argumenta que la codificación axial corresponde a una categorización que permite clasificar, ordenar y dar sentido a la información, en función de los objetivos trazados. Para realizar esta actividad se utilizó como insumo la codificación abierta, a partir de la transcripción de las entrevistas practicadas, obteniendo como resultado la siguiente tabla.

Tabla 3. Elementos de la codificación axial

\begin{tabular}{lcc}
\hline \multicolumn{1}{c}{ Nombre } & Archivos & Referencias \\
\hline COMUNICACIÓN & 42 & 133 \\
DESEMPEÑOS ACADÉMICOS & 14 & 127 \\
EMPATÍA & 42 & 108 \\
FACTORES DE VULNERABILIDAD & 22 & 98 \\
FACTORES PROTECTORES & 41 & 269 \\
RUTINAS ESCOLARES & 42 & 101 \\
TIEMPO & 42 & 63 \\
TIPOLOGIA FAMILIAR & 14 & 14 \\
\hline
\end{tabular}

Fuente. Elaboración propia.

Figura 3. Dimensiones de la codificación del proyecto

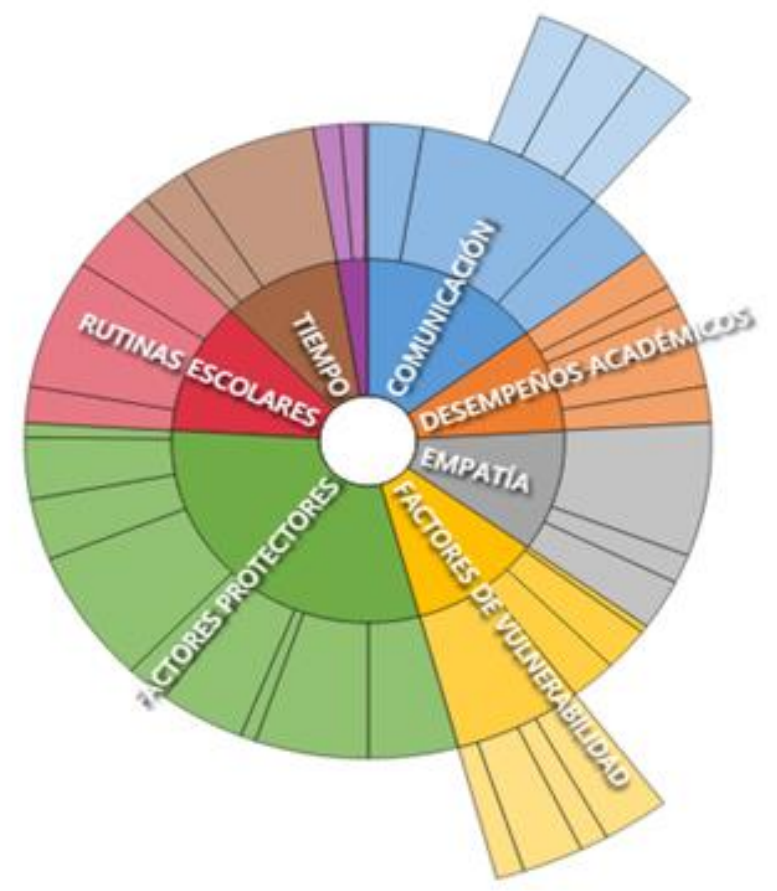

Nota. Elaboración propia

Se realizó inicialmente un análisis de los resultados obtenidos en las 14 encuestas semiestructuradas practicadas a los estudiantes, padres de familia y docente, en conjunto 
con la revisión documental del registro de desempeños académicos alcanzados en el Año lectivo 2020, encontrando lo siguiente:

Desempeño Superior: En esta subcategoría tomando los resultados de la figura 4, se identificó que 10 de los 14 los estudiantes se destacaron integralmente por obtener al menos un desempeño "Superior" en su rendimiento escolar, y que 4 estudiantes no obtuvieron ningún desempeño superior durante todo el año lectivo, es decir una ponderación máxima del $16 \%$ y mínima del $1.2 \%$.

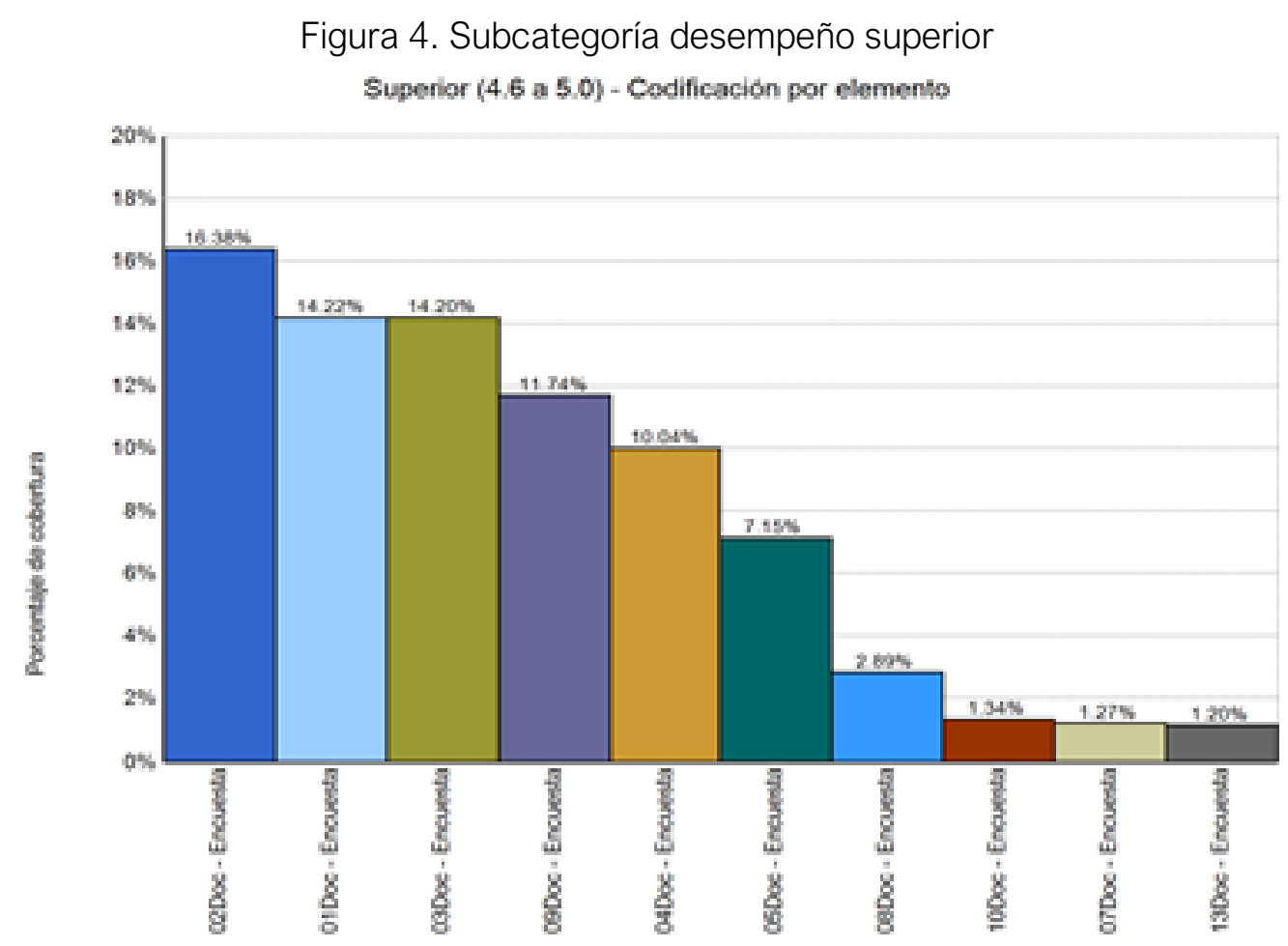

Fuente. Elaboración propia.

Al efectuar la triangulación de datos con el método propuesto y con apoyo de la herramienta mapa jerarquizante se relacionan: a) la unidad de análisis o sujeto de estudio, b) los elementos de la codificación axial y c) los desempeños "Superior"; emitiendo como resultado un informe interesante que denota de manera escalonada el nivel de influencia del entorno donde el niño interactúa, y a medida que baja el nivel de desempeño cambian los contextos que influyen en el éxito escolar como lo muestra la figura 4 para el caso de los niños con desempeño "Superior", donde el orden de priorización de las categorías según la importancia que las familias dieron a las siguientes categorías:

- $\quad$ Factores protectores (color verde)

- $\quad$ La comunicación (azul),

- Las rutinas escolares (rojo),

- La empatía (gris), el

- $\quad$ El desempeño académico (naranja),

- $\quad$ El tiempo (café) 
- $\quad$ Factores de vulneración (amarillo)

- Tipología familiar (violeta).

Figura 5. Mapa jerárquico - elemento desempeño superior

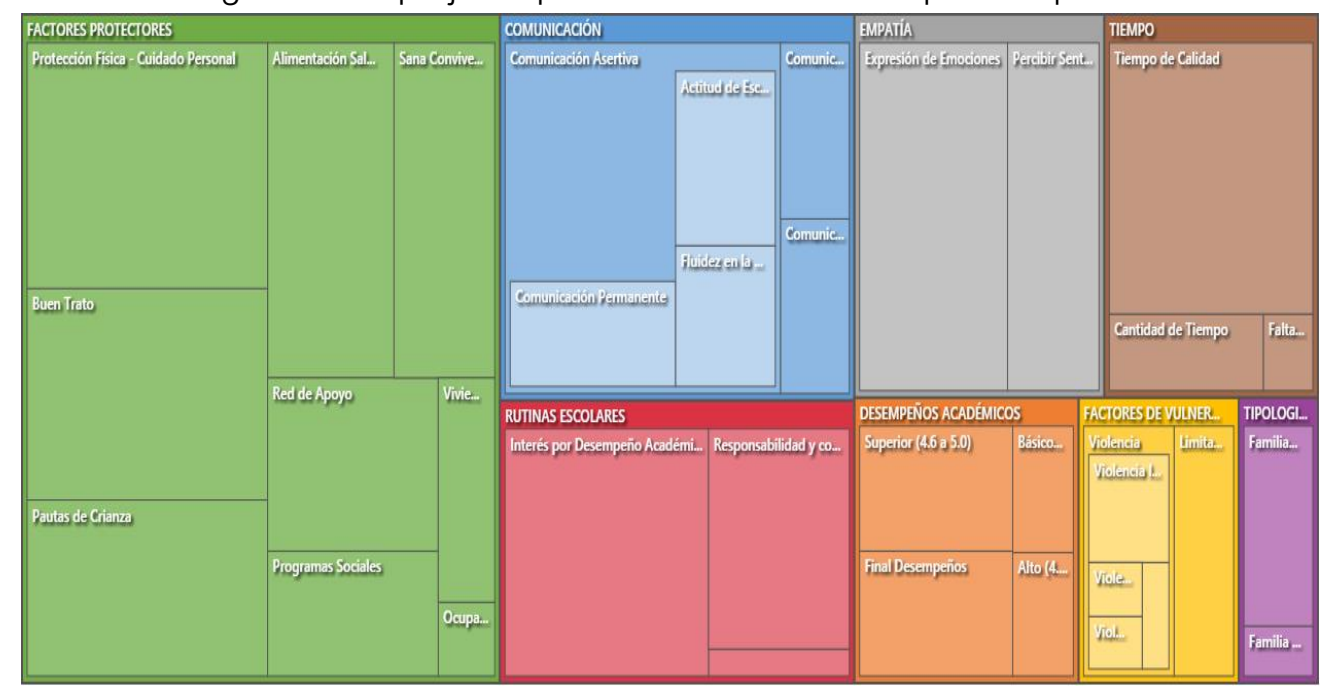

Fuente. Elaboración propia.

Desempeño Alto: En esta subcategoría se identificó que 11 de los 14 los estudiantes se destacaron integralmente por obtener al menos un desempeño "Alto", en su rendimiento escolar con una ponderación máxima del $11,33 \%$ y mínima del $0.40 \%$, y que 3 estudiantes no obtuvieron ningún desempeño Alto, como se puede evidenciar en la figura 5.

Figura 6. Subcategoría desempeño alto

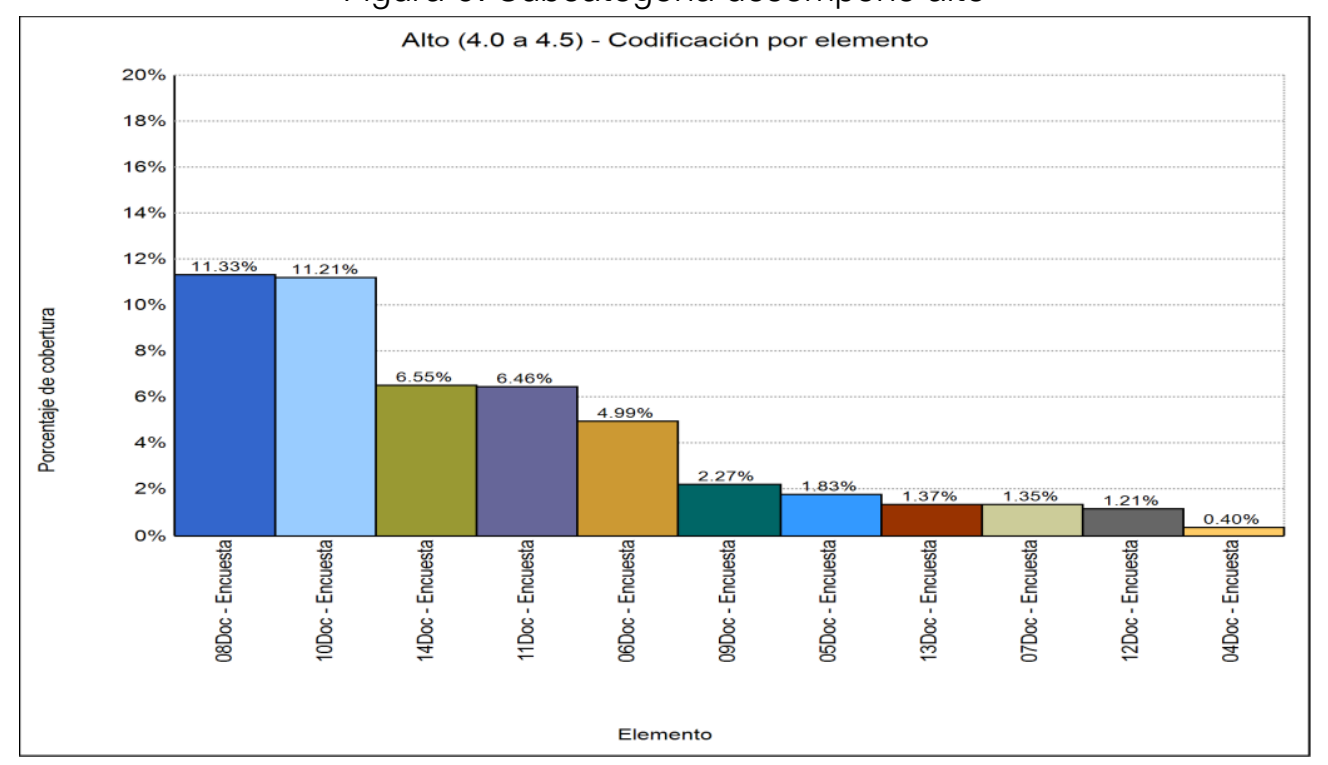

Fuente. Elaboración propia.

Al efectuar una triangulación de datos con la herramienta mapa jerarquizante entre: a) la unidad de análisis o sujeto de estudio, b) los elementos de la codificación axial y c) los desempeños "Alto"; emite como resultado un informe que denota de manera escalonada (hacer comparaciones con los cuadros de acuerdo a su color) el nivel de influencia del 
entorno donde el niño interactúa, y a medida que baja el nivel de desempeño cambian los entornos que afectan sus desempeños como se observa en la figura 5 donde se indica el orden de priorización de las categorías, según su importancia: factores protectores, comunicación, rutinas escolares, factores de vulnerabilidad, empatía, tiempo, desempeños académicos y tipología familiar.

\section{Figura 7. Mapa jerárquico - elemento desempeño alto}

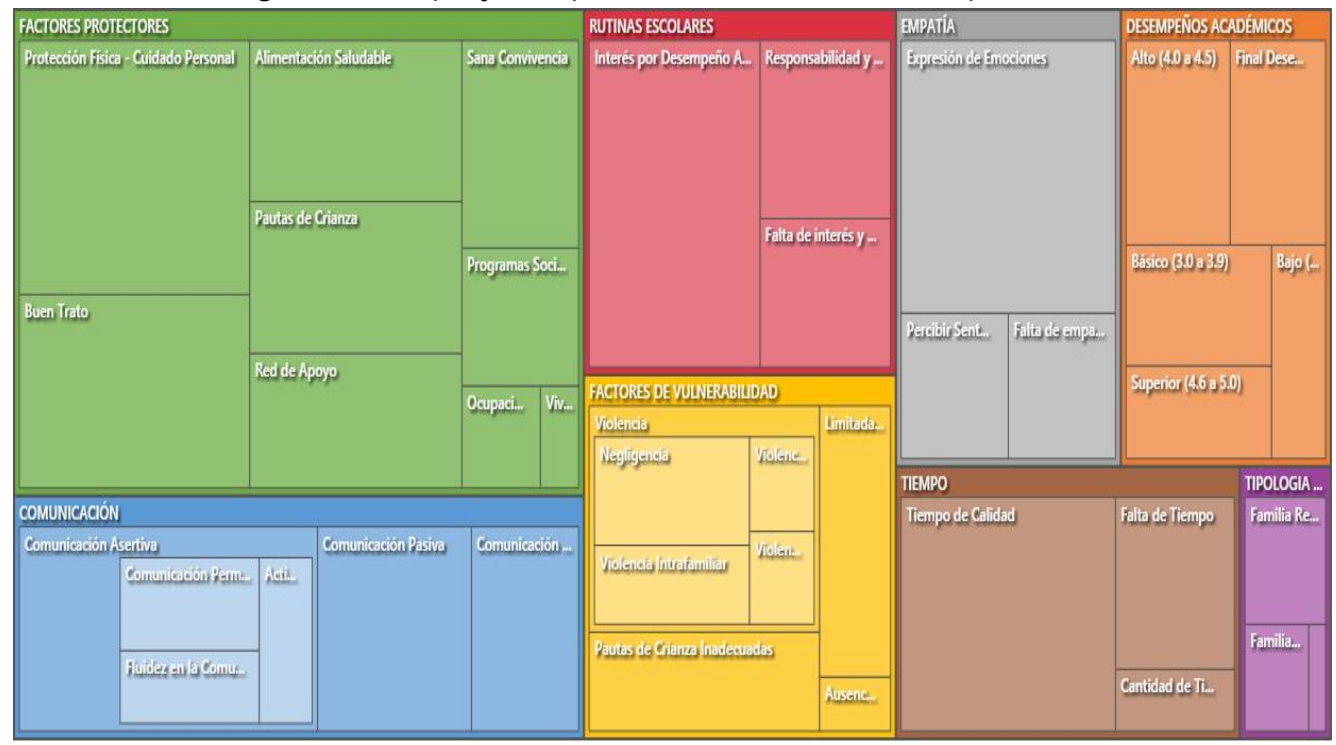

Fuente. Elaboración propia.

Desempeño Básico: En esta subcategoría se identificó que 10 de los 14 los estudiantes de los grados tercero, cuarto y quinto del aula multigrado se destacaron integralmente por obtener al menos un desempeño básico en su rendimiento escolar con una ponderación máxima del $9.96 \%$ y mínima del $1.74 \%$, y que 4 estudiantes no obtuvieron ningún desempeño "Básico", como se puede evidenciar en la figura 3 que emite el Software NVIVO12.

Figura 8. Subcategoría desempeño bajo

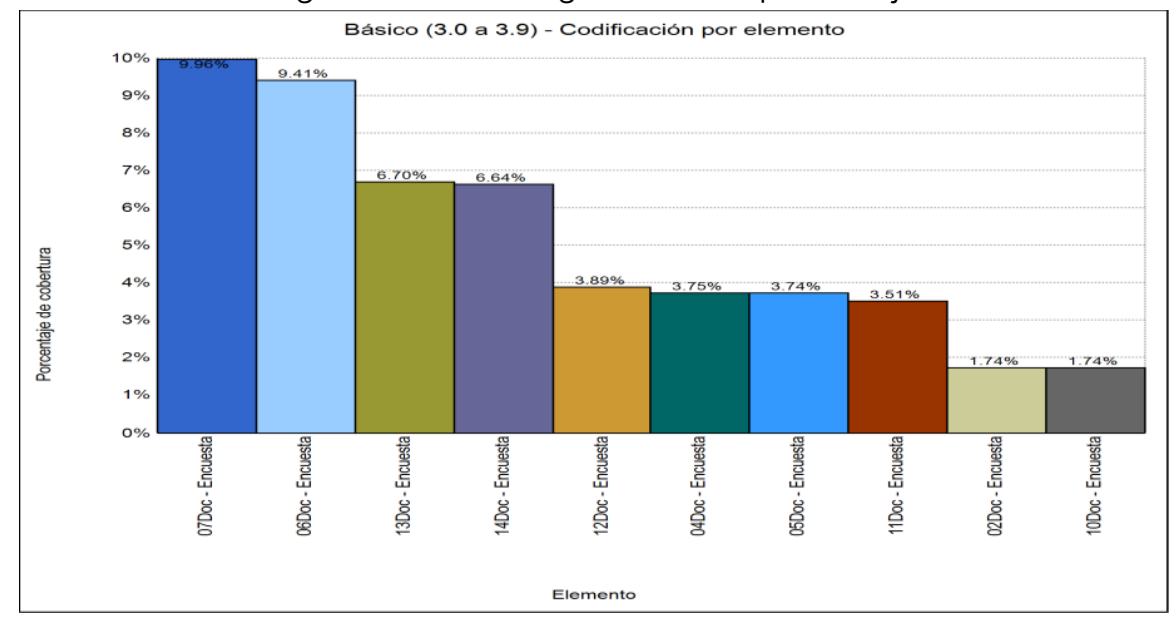

Fuente. Elaboración propia.

Al efectuar una triangulación de datos con la herramienta mapa jerarquizante entre: a) la unidad de análisis o sujeto de estudio, b) los elementos de la codificación axial y c) los 
desempeños "Básico"; emite como resultado un informe que denota de manera escalonada (hacer comparaciones con los cuadros de acuerdo a su color) el nivel de influencia que el entorno donde el niño interactúa, y a medida que baja el nivel de desempeño cambian los entornos que influyen en el aprendizaje como se observa en la figura 7 donde se indica el orden de priorización de las categorías, según su importancia: factores protectores, comunicación, factores de vulnerabilidad, desempeño académico, empatía, rutinas escolares, tiempo y tipología familiar.

Figura 9. Mapa jerárquico - elemento desempeño básico

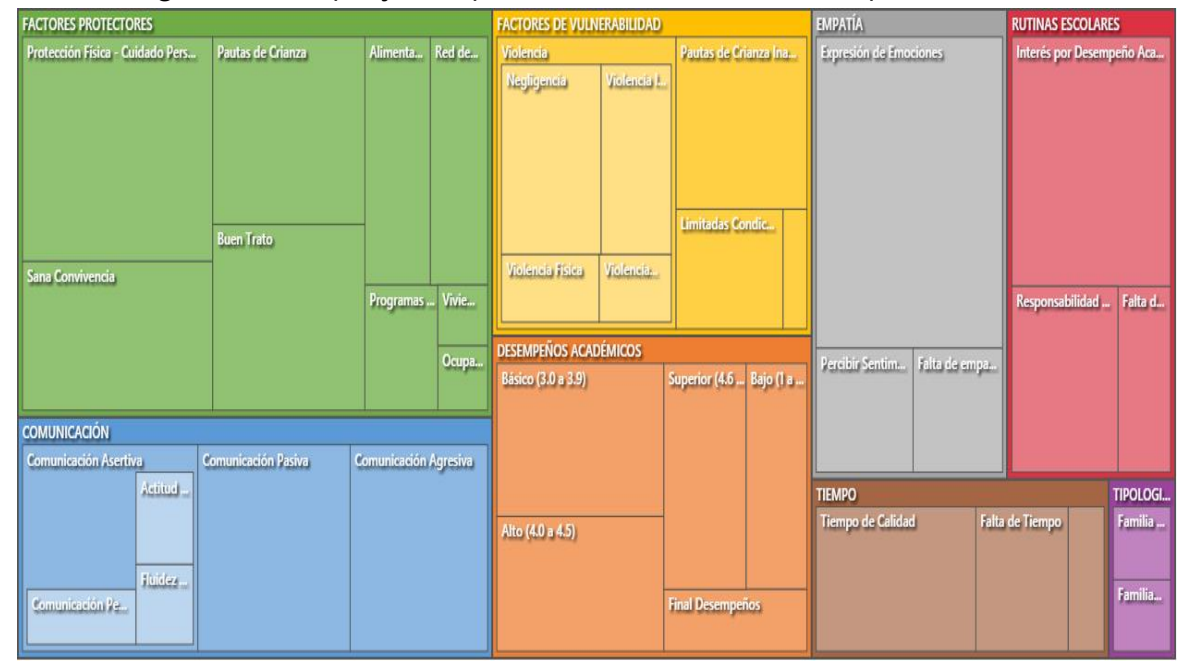

Fuente. Elaboración propia.

Desempeño Bajo: En esta subcategoría se identificó que 6 de los 14 los estudiantes de los grados tercero, cuarto y quinto del aula multigrado se destacaron integralmente por obtener al menos un desempeño "bajo", en su rendimiento escolar con una ponderación máxima del $13.75 \%$ y mínima del $1.75 \%$ y que 4 estudiantes no obtuvieron ningún desempeño "Básico", como se puede evidenciar en la figura 8 que emite el Software NVIVO12.

Figura 10. Subcategoría desempeño bajo

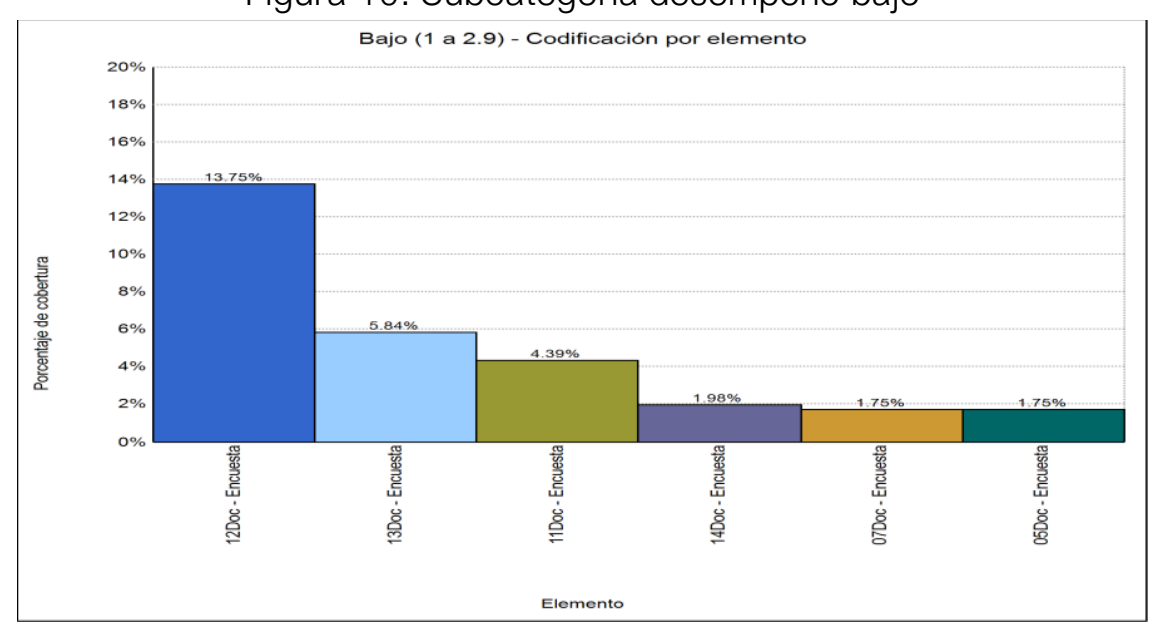

Fuente. Elaboración propia. 
Al efectuar una triangulación de datos con la herramienta mapa jerarquizante entre: a) la unidad de análisis o sujeto de estudio, b) los elementos de la codificación axial y c) los desempeños "Bajo"; emite como resultado un informe que denota de manera escalonada (hacer comparaciones con los cuadros de acuerdo a su color) el nivel de influencia que el entorno donde el niño interactúa, y a medida que baja el nivel de desempeño cambian los entornos que afectan su rendimiento académico como se observa en la figura 9 donde se indica el orden de priorización de las categorías, según su importancia: factores protectores, de vulnerabilidad, comunicación, rutinas escolares, empatía, tiempo, desempeños académicos y tipología familiar.

Figura 11. Mapa jerárquico - elemento desempeño bajo

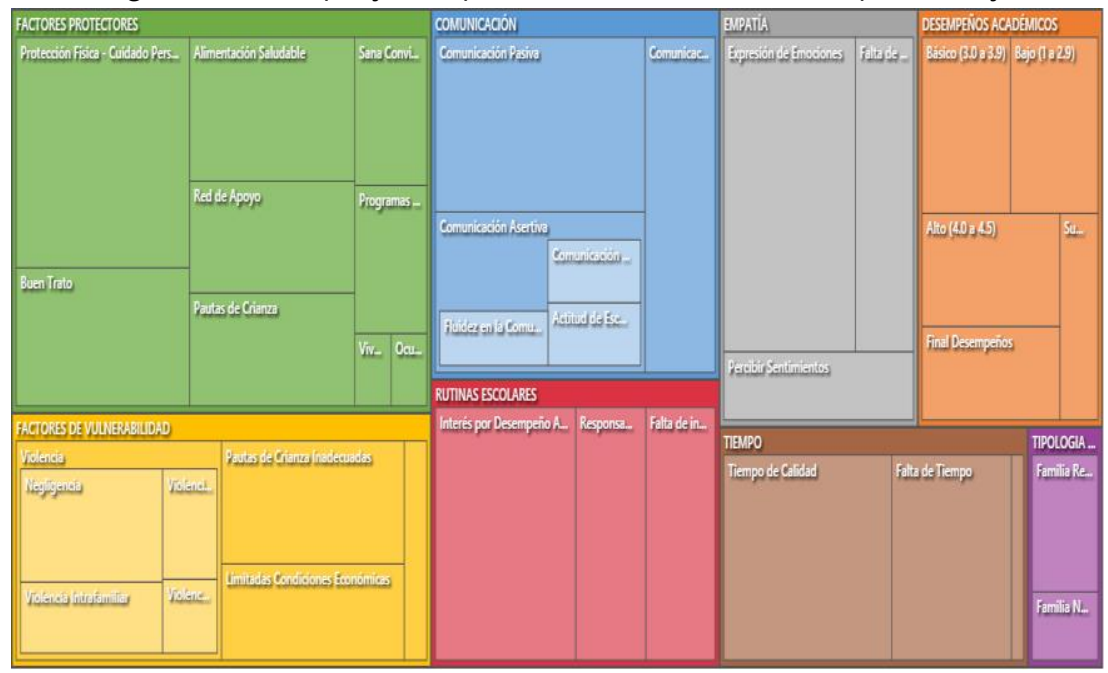

Fuente. Elaboración propia.

Aquí podemos hacer una breve comparación y detallar claramente las diferencias encontradas en cada una de las categorías de acuerdo al tipo de desempeño, como el caso principalmente de los factores de vulneración visualizado de color amarillo ocupando un segundo lugar en las familias de los niños que obtuvieron desempeño "bajo"; mientras que los que obtuvieron desempeño "Superior" ocuparon un séptimo lugar con mínima incidencia; del mismo modo se logró detallar las demás categorías y su nivel de incidencia de acuerdo al tipo de desempeño.

\section{DISCUSIÓN}

Desde el punto de vista constructivista el comportamiento humano no puede ser comprendido y apreciado por fuera del contexto en el que ocurre; es decir por fuera del medio familiar, social, psicológico, cultural y económico en el cual viven las personas, es por ello por lo que a partir de los hallazgos encontrados se puede asegurar que los vínculos afectivos son factores que influyen en el desempeño académico de los estudiantes en edad infantil entre 8 y 11 años. Estos resultados guardan relación con lo que sostiene el pedagogo constructivista Pestalozzi cuando habla de la importancia que representa el ambiente familiar en la formación de la personalidad de los niños. Amor, confianza y gratitud son 
valores, decía, por los que debe velar la educación. Aquí es importante hacer referencia a que el ser humano inicia su proceso de aprendizaje, incluso desde el momento de la concepción, lo desarrolla en el seno familiar gracias a la interacción diaria con sus padres y hermanos y lo amplia en la escuela con sus amigos y profesores.

La caracterización realizada en la relación Paternofilial y su influencia frente al desempeño académico de 14 niños y niñas en edades de 8 a 11 años de la institución educativa Rural San Antonio del Municipio de San Francisco Putumayo revela que aunque existen vínculos afectivos en la mayoría de las familias, hay algunas de estas que presentan particularidades y/o dificultades en su interacción, y son los niños de éstas familias los que principalmente evidencian un desempeño académico regular (6 Niños), los cuales de acuerdo a los resultados de las valoraciones del año lectivo 2020 están relacionados con familias de tipología reconstruida (madrasta y/o - padrastro) y/o monoparentales bajo la figura de la Madre o del padre como Cabeza de Hogar, quienes cuentan con poco tiempo para compartir y/o participar de las actividades con su hijo; en contraposición a lo anterior, se encuentra que la mayoría cuentan con una buena dinámica familiar, producto de una sana convivencia con los padres o cuidadores; otra de las categorías que sobresalió , fue las dificultades en el acatar y establecer normas y reglas en el hogar, la cantidad y calidad de tiempo compartido, al igual que la capacidad de resolución de conflictos en las actividades cotidianas, las cuales influyen directamente en el desempeño académico de los estudiantes. Otra de las unidades de análisis que representa una diferencia en el rendimiento escolar, hace referencia a las familias con modelos rígidos de autoridad, imposición de normas, presencia de violencia verbal e intrafamiliar, ausencia del padre biológico en la crianza y cuidado, creando factores de vulnerabilidad y lesionando representativamente el bienestar de la infancia. De igual forma, otros factores que influyen positivamente a pesar de que cada familia atraviesa distintas particularidades es el respeto por las diferencias de pensamiento, la democracia, el dialogo asertivo, el compartir tiempo de calidad, la participación de los padres en su proceso académico, la comunicación, los valores, normas que infunden en el hogar y el nivel económico; estos permiten superar el bajo rendimiento académico.

De acuerdo con el objetivo planteado y los resultados obtenidos, se pudo constatar que existen otros factores que inciden negativamente en el desarrollo del aprendizaje y desempeño académico como la desintegración familiar donde el niño-niña es criado por la madre o cualquier otro familiar, la falta de comunicación con sus hijos, el desinterés y la poca motivación de los padres hacia la integración en el proceso académico del niño, además del ingreso económico bajo en el hogar. Confirmando así, lo expuesto por el psicólogo Gil, creador de la teoría del apego al referir que "son las relaciones afectivas positivas que nos ayudan a crecer como personas. Este ha de ser recíproco complementario, bidireccional, a saber, implica dar y recibir" (2007). Afirma además que "las caricias, besos, el afecto, la escucha, el respeto mutuo y el cariño ayudan a la evolución, desarrollo y madurez física, intelectual y emocional" (2007).

\section{CONCLUSIONES}


La cimentación de los vínculos en la relación paternofilial se fabrica a partir de los signos y representación naciente en las relaciones en tiempo y espacio. Con base a la categorización de las unidades de análisis se puede evidenciar que a pesar de que en algunos casos hay familias que fortalecen sus vínculos gracias a la comunicación, el compartir diario, la identificación de emociones, la escucha activa, el dialogo asertivo, el cumplimiento de normas y límites, como factor fundamental de su desarrollo social, emocional, físico y cognitivo de los niños sujetos de estudio, hay otras familias que tienen debilidades en su estructura y dinámica familiar que afectan de diferente manera el desarrollo del aprendizaje y desempeño académico en la etapa de la infancia, esto en razón a distintos factores y particularidades de cada familia que permiten desarrollar en cada niño, niña diferentes niveles de afrontamiento.

Se determinó de manera individualizada y grupal, cómo influyen y afectan los diferentes entornos respecto a los vínculos afectivos y el desempeño académico de 14 estudiantes de los grados tercero, cuarto y quinto, caracterizándose mayormente la negligencia, las condiciones socioeconómicas, las inadecuadas pautas de crianza y conformación familiar de tipo reconstruida como principales factores de riesgos amenazantes y/o vulnerantes de derechos de los niños, niñas y adolescentes, en línea escalonada con el desempeño escolar.

A pesar de que los seres humanos desde siempre han establecido relaciones emocionales caracterizadas por la búsqueda de cercanía, el vínculo afectivo es un tema muy poco estudiado, más aún cuando se sabe que el afecto es generador de impactos positivos en los niños y las niñas y que esos efectos se extienden hasta la edad adulta. Cuando una persona evidencia una vinculación afectiva sana le es más fácil adaptarse a las exigencias de la sociedad actual pues hoy en día se considera que el éxito depende más de la inteligencia emocional que la racional. La sociedad contemporánea requiere de seres humanos seguros, con relaciones saludables, capacidad de amar, aceptarse así mismo, a los demás y ser feliz. Ahora más que nunca se hace necesario saber cómo es la relación familiar, descubrir sus puntos críticos y desde la institucionalidad abordar estrategias que generen aprendizaje continuo de los involucrados en la educación de los más pequeños.

\section{REFERENCIAS}

Carretero, M. y Limón, M. (1993). Constructivismo y educación. pp. 62-63. 153-167

Gil, M. (2007). La familia. Claves para una correcta gestión de las personas y situaciones familiares. Barcelona: Amat

Hernández, R., Fernández, C., Baptista, P. (2010). Metodología de la Investigación. Sexta edición. Editorial Mc Graw Hill. México.

Hernández, R., Fernández, C., y Baptista, P. (2014) Metodología de investigación. $6^{\circ}$ edición. México: Ed. Mc Graw Hill. 
Hernández Sampieri, R., Fernández Collado, C., \& Baptista Lucio, P. (2014). Metodología de la investigación: Roberto Hernández Sampieri, Carlos Fernández Collado y Pilar Baptista Lucio. 6a. edición

Morón Marchena, J. A., Pérez-Pérez, I., Pedrero García, E., (2017). Educación para la Salud y prevención de riesgos psicosociales. En adolescentes y jóvenes. Madrid: Narcea, S.A. de Ediciones.

Perry, Chat. (1998) "Proceses of case study por postgraduate research in marketin", european jornal of marketing 9/10 785802

QSR International. (2014). NVIVO 10 for Windows. Primeros pasos. QSR International.

Strauss, Anselm y Corbin Juliet (2002). Bases de la investigación cualitativa. Técnicas y procedimientos para desarrollar la teoría fundamentada. Editorial Universidad de Antioquia. Colombia.

Yin, R. (2009), Case Study Research. London: Sage

Yin, R. (1994) Case study research: Design and methods. California: Sage. 